\title{
Explore or reset? Pupil diameter transiently increases in self-chosen switches between cognitive labor and leisure in either direction
}

\author{
Johannes Algermissen $^{1,2}$ (D) Erik Bijleveld $^{2} \cdot$ Nils B. Jostmann $^{3} \cdot$ Rob W. Holland $^{2}$
}

Published online: 17 June 2019

(C) The Author(s) 2019

\begin{abstract}
When people invest effort in cognitive work, they often keep an eye open for rewarding alternative activities. Previous research suggests that the norepinephrine (NE) system regulates such trade-offs between exploitation of the current task and exploration of alternative possibilities. We examined the possibility that the NE system is involved in another trade-off, i.e., the trade-off between cognitive labor and leisure. We conducted two pre-registered studies (total $N=62$ ) in which participants freely chose to perform either a paid 2-back task (labor) versus a non-paid task (leisure), while we tracked their pupil diameter-which is an indicator of the state of the NE system. In both studies, consistent with prior work, we found (a) increases in pupil baseline and (b) decreases in pupil dilation when participants switched from labor to leisure. Unexpectedly, we found the same pattern when participants switched from leisure back to labor. Both increases in pupil baseline and decreases in pupil dilation were short-lived. Collectively, these results are more consistent with a role of norepinephrine in reorienting attention and task switching, as suggested by network reset theory, than with a role in motivation, as suggested by adaptive gain theory.
\end{abstract}

Keywords Decision-making $\cdot$ Cognitive effort $\cdot$ Exploration-exploitation trade-off $\cdot$ Motivation $\cdot$ Norepinephrine $\cdot$ Pupillometry

\section{Introduction}

In their pursuit of rewards, such as food, all organisms are continuously confronted with two choices: continue exploiting their current location to harvest rewards, or leave their current place to explore the environment for potentially more attractive alternatives. These two strategies, called exploitation and exploration, can both contribute to maximizing rewards in the long-term. When organisms exploit, they aim to maximize rewards by sticking with the activity that they believe yields the highest payoff at the moment. When organisms explore, they aim to maximize rewards by gathering

Electronic supplementary material The online version of this article (https://doi.org/10.3758/s13415-019-00727-x) contains supplementary material, which is available to authorized users.

Johannes Algermissen

j.algermissen@donders.ru.nl

1 Donders Centre for Brain, Cognition and Behavior, Radboud University, Montessorilaan 3 B.08.25, 6525 HR Nijmegen, the Netherlands

2 Radboud University, Nijmegen, the Netherlands

3 University of Amsterdam, Amsterdam, the Netherlands information on whether other activities yield higher returns. This fundamental dilemma between exploitation and exploration plays a central role in foraging models, which have a rich history in behavioral ecology (Charnov, 1976). Foraging models have proven valuable in various fields, including artificial intelligence, psychology, and neuroscience (Calhoun \& Hayden, 2015; Hayden, 2018; Hills, Todd, Lazer, Redish, \& Couzin, 2015), and have recently been suggested as a general framework for understanding value-based choice (Hayden, 2018; Hunt \& Hayden, 2017; Rushworth, Kolling, Sallet, \& Mars, 2012).

A limitation of previous foraging experiments with humans is that these experiments did not account for the effort costs of different activities (Constantino \& Daw, 2015; Kolling, Behrens, Mars, \& Rushworth, 2012). In real life, obtaining higher rewards often requires higher effort exertion (e.g., longer, faster, or more intense performance in sports), and thus higher effort is often strategically invested for higher reward prospects (Bijleveld, Custers, \& Aarts, 2009, 2012). Similar to reward pursuit in foraging, also physical (Meyniel, Sergent, Rigoux, Daunizeau, \& Pessiglione, 2013) and mental (Kool \& Botvinick, 2014) effort mobilization are not constant over time. Instead, effort mobilization is interrupted by breaks that might serve the recreation from effort expansion (Jett \& George, 2003). It thus makes sense to conceptualize decisions 
for rewards not just as a trade-off between exploration and exploitation but also as a trade-off between cognitive labor (i.e., exerting effort to attain a reward) and cognitive leisure (i.e., performing a nondemanding and nonprofitable activity, such as relaxing; Kool \& Botvinick, 2014, 2018). Note that labor and leisure are conceptualized in relative terms, such that a task is a leisure task if it provides relief from effort mobilization in another (labor) task; no task is intrinsically labor or leisure.

It is important to note that these two dilemmasexploration versus exploitation and labor versus leisure - are conceptually independent. After all, exploitation versus exploration concerns the question of how to maximize reward, whereas labor versus leisure concerns the question of how to optimally allocate effort. The exploration-exploitation dilemma emerges when people face a choice between (a form of) short-term reward pursuit and (a form of) information gathering, whereas the labor-leisure dilemma emerges when people face a choice between some high-effort activity and some activity that provides relief. Because of their independence, the exploration-exploitation and labor-leisure trade-offs do not directly map onto each other. That is, labor activities can sometimes be exploitative (e.g., when working on a spreadsheet helps to make progress to a work-related goal, and thus, is continued), but at other times is exploratory (e.g., when working on a spreadsheet is chosen as a potentially rewarding distraction from some other activity). Similarly, leisure activities can be exploitative (e.g., when talking to colleagues at work is rewarding, and thus, is continued) but also exploratory (e.g., when talking to colleagues is chosen as a potentially rewarding distraction from some other activity).

In this research, we examined the particular case in which people are faced with the choice between high-effort cognitive work that yields a higher expected reward (i.e., labor) and taking a low-effort break that yields a lower expected reward (leisure). In our view, this case is prototypical of many real-life decisions under uncertainty. In such cases, labor often may co-occur with exploitation (since labor tasks reliably yield external rewards), and leisure often may coccur with exploration (since leisure tasks yield no external reward, but provide opportunities to detect other, more intrinsic rewards, e.g., encouraging social interactions). Our research concerns the particular cases in which this mapping holds and is agnostic about other cases in which this mapping is reversed or more dynamic (e.g., when this mapping changes over time because the rewards associated with labor and leisure change over time). By studying these particular cases, we examined a potential connection between the two tradeoffs. In particular, using a labor-leisure paradigm, we investigated whether the neural mechanisms that people seem to use to balance exploitation and exploration (i.e., the locuscoeruleus norepinephrine system) also may be used for trading off cognitive labor and leisure.
Our research exceeds prior work in two ways. First, we used a dual-task paradigm with two qualitatively different tasks. We chose to use this paradigm to model decisions in real life, where labor (e.g., working on a spreadsheet) usually comprises a vastly different activity than leisure (e.g., talking to colleagues). Second, we examined transitions between labor and leisure in both directions. That is, we not only examined decisions to take breaks (while working), but also examined when people decided to start working again (after taking a break). An important feature of our studies is that analysis plans were pre-registered before data collection. In what follows, we introduce the major biological theory linking the exploration-exploitation trade-off to neural processes, describe previous human research supporting this theory, and explain why our paradigm allows for a more comprehensive investigation of NE levels when humans switch between labor and leisure.

A major theory linking the trade-off between exploration and exploitation to neural mechanisms is adaptive gain theory, which connects these behavioral states to qualitatively different neural states of the locus coeruleus-norepinephrine (LCNE) system (Aston-Jones \& Cohen, 2005; Cohen, McClure, $\& \mathrm{Yu}, 2007)$. According to adaptive gain theory, exploitation is driven by a phasic mode of locus coeruleus (LC) activity. In the phasic mode, baseline norepinephrine (NE) levels in the $\mathrm{LC}$ are moderate, but bursts of NE release occur in response to task-related stimuli. Given that NE release in cortical areas increases neural responsivity to incoming information (Berridge \& Waterhouse, 2003; Servan-Schreiber, Printz, \& Cohen, 1990), this pattern is likely adaptive: it helps animals to process task-relevant information. In contrast, exploration is driven by a tonic mode of LC activity. In the tonic mode, baseline NE levels are chronically elevated, and bursts of NE (in response to task-relevant stimuli) are attenuated or even absent. It has been suggested that this pattern may widen attention, allowing people to better detect task-irrelevant-but potentially rewarding - stimuli (Cohen et al., 2007).

Importantly, NE levels are correlated with pupil diameter in both monkeys (Joshi, Li, Kalwani, \& Gold, 2016; Rajkowski, Kubiak, \& Aston-Jones, 1994) and humans (Murphy, O'Connell, O'Sullivan, Robertson, \& Balsters, 2014; Murphy, Robertson, Balsters, \& O'Connell, 2011). For example, in line with predictions from adaptive gain theory, previous research found that pupil diameter covaried with task engagement and disengagement in an auditory discrimination task (Gilzenrat, Nieuwenhuis, Jepma, \& Cohen, 2010). In this task, participants judged which of two tones had a higher pitch. Task difficulty continuously increased while rewards decreased with every error a participant made. At any time, participants could reset task settings by pressing an "escape" button, which the authors interpreted as exploration behavior. Changes in pupil dilation also were observed in transitions between bandit gambling machines (Jepma \& Nieuwenhuis, 
2011) and in a task that required people to solve Raven's Matrices (Hayes \& Petrov, 2016). Overall, in these studies, exploitation was characterized by relatively low baseline pupil diameter, combined with large pupil dilations in response to task stimuli. By contrast, exploration was characterized by high baselines and smaller dilations in response to taskrelevant stimuli.

While the studies described in the previous paragraph link the LC-NE system, and particularly pupil diameter as an observable correlate, to exploration-exploitation dilemmas, it is not yet clear whether the LC-NE system is involved in people's decisions to switch between labor and leisure. After all, prior studies operationalized exploration as brief transitions between extended phases of exploitation, making it impossible to disentangle disengagement from the previous task versus reengagement in the next task. Also, these studies manipulated task payoff and difficulty, thus incentivizing all participants to start exploration at a defined moment, instead of keeping the environment constant and tracking individual differences in participants' natural drive for exploration. In contrast, we studied participants' self-directed decisions to take a break (vs. to continue working), which are arguably similar in structure as exploration-exploitation dilemmas, because agents have to decide whether (a) to stay with a current activity and its payoff or (b) to quit this activity and explore the environment for more rewarding alternatives - at the risk of wasting time and foregoing rewards. Indeed, in research on fatigue and effort, the LC-NE system has been mentioned as a candidate mechanism for understanding labor-leisure transitions (Inzlicht, Schmeichel, \& Macrae, 2014; Kurzban, Duckworth, Kable, \& Myers, 2013). Yet, to our knowledge, this possibility has not been tested.

\section{Present research}

Based on adaptive gain theory, we hypothesized that laborto-leisure transitions (i.e., decisions to take a break from work) are preceded by (a) increases in pupil baseline and (b) decreases in pupil dilation. For leisure-to-labor transitions (i.e., decisions to start working after taking a break), predictions are somewhat less straightforward. On the one hand, based on adaptive gain theory, we would predict that baselines stay high during the leisure phase to ensure a broadened attention that facilitates the detection of alternative activities. However, when switching back to labor, people need to focus on the labor task only, so that (a) pupil baseline should decrease and (b) pupil dilations should increase again. On the other hand, one also could pose that leisure-to-labor transitions are not different from labor-toleisure transitions in that they constitute a case of taskswitching. A role of NE in task switching has been suggested by network reset theory (Bouret \& Sara, 2005; Sara
\& Bouret, 2012), which suggests that NE is primarily released when people detect unexpected changes in the environment. When these changes happen, NE promotes reorientation towards stimuli that have become relevant in the new task environment, facilitating adaptation to new demands. So far, it has not been explored whether NE could have a similar function in humans' self-directed decisions to switch tasks. If this was the case, we would expect (a) increases in pupil baseline and (b) decreases in pupil dilation preceding both labor-to-leisure and leisureto-labor transitions, because network reset theory assumes NE processes to be independent of the activity performed previously and the activity to-be-performed after the switch. By investigating leisure-to-labor transitions, our research directly tested adaptive gain theory and network reset theory against each other.

We operationalized labor as a 2-back memory task and leisure as an attractiveness rating task, following a paradigm by Kool and Botvinick (2014). We employed both linear and additive mixed models, which allowed us to more closely examine the onset and duration of pupil shifts. To shed light on the functional role of the processes underlying pupil changes, we conducted exploratory analyses in which we correlated the magnitude of pupil changes with individual differences in self-reported procrastination and action orientation in everyday life. To this end, we used well-validated questionnaires (Kuhl, 1994; Kuhl \& Fuhrmann, 1998; Steel, 2010). We chose personality dimensions that reflected how readily people engaged into effortful tasks and how they dealt with fatigue following from effort. We explored whether people who successfully dealt with these challenges in everyday life would show a different pupil pattern around switches than people who had problems with initiating or maintaining effort mobilization. These analyses were exploratory and served hypothesis-generation for future studies.

We report the methods and results for Studies 1 and 2 together, because protocols in both studies were identical except for two brief control assessments that were administered following the main task in Study 2. In our pre-registration for Study 1, based on previous literature (Gilzenrat et al., 2010), we expected pupil shifts to occur before task switches, which would have allowed us to predict switches prospectively based on pupil diameter. ${ }^{1}$ Because our hypotheses found no evidence in the pre-registered time window, we explored them in a later time window centered around the behavioral switch. We preregistered these analyses for Study 2 to replicate our exploratory findings from Study $1 .^{2}$

\footnotetext{
${ }^{1}$ Pre-registration for Study 1: https://osf.io/ypyha/register/ 5730e99a9ad5a102c5745a8a

${ }^{2}$ Pre-registration for Study 2: https://osf.io/48kyw/register/ 5730e99a9ad5a102c5745a8a
} 


\section{Methods}

\section{Participants}

In Study 1, 35 participants completed a 50-minute study in exchange for a $€ 7.50$ voucher and an extra cash payment of up to $€ 5$, depending on their task performance. We recruited participants in the age range 17-30 years who had normal or corrected-to-normal vision (using contact lenses), understood English, and did not suffer from neurological disorders. In line with pre-registered exclusion criteria (see Supplementary Online Material S1), four participants were excluded from analyses, so that the final sample consisted of 31 participants (68\% female, $\left.M_{\text {age }}=22, S D_{\text {age }}=2.7\right)$.

In Study 2, 35 participants completed a 60-minute study in exchange for a $€ 10$ shopping voucher and an extra cash payment of up to $€ 5$. Participants were recruited from the same population as in Study 1, using the same exclusion criteria (see S2). Four participants were excluded from analyses, so that the final sample consisted of 31 participants (65\% female, $M_{\text {age }}=22, S D_{\text {age }}=3.1$ ). Studies were approved by the local ethics review board.

\section{Procedure}

Participants were welcomed in a room equipped with a stationary SMI iView X infrared eye-tracker (SensoMotoric Instruments, Teltow, Germany) sampling at $500 \mathrm{~Hz}$. We placed this device's chin rest 72 -cm away from a 24-inch monitor, on which the task stimuli were presented (using a script programmed in PsychoPy; Peirce, 2007). After they were seated, participants first completed a 9-point calibration of the eye-tracking device, followed by a 10-min practice phase, in which participants were familiarized with the labor and leisure tasks (which were first practiced separately), but also with labor-to-leisure and leisure-to-labor switches.

Next, participants completed the test phase, which took $25 \mathrm{~min}$ and consisted of 500 trials. During both the practice and test phases, pupil size of their dominant eye was tracked. In Study 2, additionally, participants completed two control assessments of 80 trials each to exclude alternative interpretations of the findings of Study 1. Afterwards, in both studies, participants rated both tasks on the dimensions work and fun using a 7-point scale. We assumed that the work ratings reflected whether participants perceived the labor task as more effortful than the leisure task, which would mean that our task successfully fulfilled the criterion for a labor-leisure trade-off. In contrast, the fun ratings assessed participants' intrinsic motivation (opposed to the extrinsic monetary incentives) to do a task. These fun ratings were important to exclude the possibility that participants performed the labor task not because it returned monetary rewards, but because it was more fun than the leisure task. If participants were motivated by fun instead of maximizing rewards, neural mechanisms different from those designed to deal with exploration-exploitation dilemmas might have been involved. Afterwards, participants filled in questionnaires assessing their procrastination tendency and action versus state orientation. Finally, they were debriefed and paid.

\section{Labor-leisure task}

During the test phase, in each trial, participants could choose between performing an effortful 2-back task (labor task) or an easy attractiveness-rating task (leisure task; adapted from Kool \& Botvinick, 2014). For each trial they spent on the labor task, they received 1-cent extra cash payment. It was announced in advance that participants with accuracy levels below $75 \%$ in the labor task would receive no extra payment but that previous participants were able to easily achieve this level if they tried hard. This threshold was implemented to discourage participants from resting during the labor task and encourage switching to the leisure task in case they felt like taking a break. Participants' responses during the leisure task did not affect their monetary payout, allowing for an actual break from paid work.

Participants selected and performed tasks using a joystick. Specifically, participants selected tasks by moving the joystick sideways. When the joystick was moved to the left (or right; counterbalanced), participants performed the labor task; when moved to the right (or left; counterbalanced), participants performed the leisure task. Both tasks were presented as labels at the upper corners of the screen ("2-back" and "attractiveness"). As a reminder, the currently selected task was surrounded by a white frame. It was only possible to switch tasks when no stimulus was displayed to prevent participants from employing the strategy of first viewing a face and then choosing which task to perform. Participants indicated responses via the single backward trigger of the joystick. We chose to use a joystick in line with the original paradigm by Kool and Botvinick (2014). Compared with a keyboard, a joystick reduces the chance that participants accidently press a different key than they intend; after all, when using a stationary eye tracking device with a chin and head rest, participants could not visually check their manual responses.

Regardless of the position of the joystick, on each trial, participants saw the following stimuli: a fixation cross (200 $\mathrm{ms})$, a mask stimulus $(800 \mathrm{~ms})$, a face $(1500 \mathrm{~ms})$, and a blank screen (400-600 ms). Figure 1 depicts the course of one trial. When participants had selected the labor task, they were required to press the joystick trigger when the current face was the same face as two faces before (i.e., when the current face was a 2-back target; in case of a nontarget, they were required to refrain from responding). When participants had selected the leisure task, they were asked to press the joystick trigger when they saw an attractive face. 


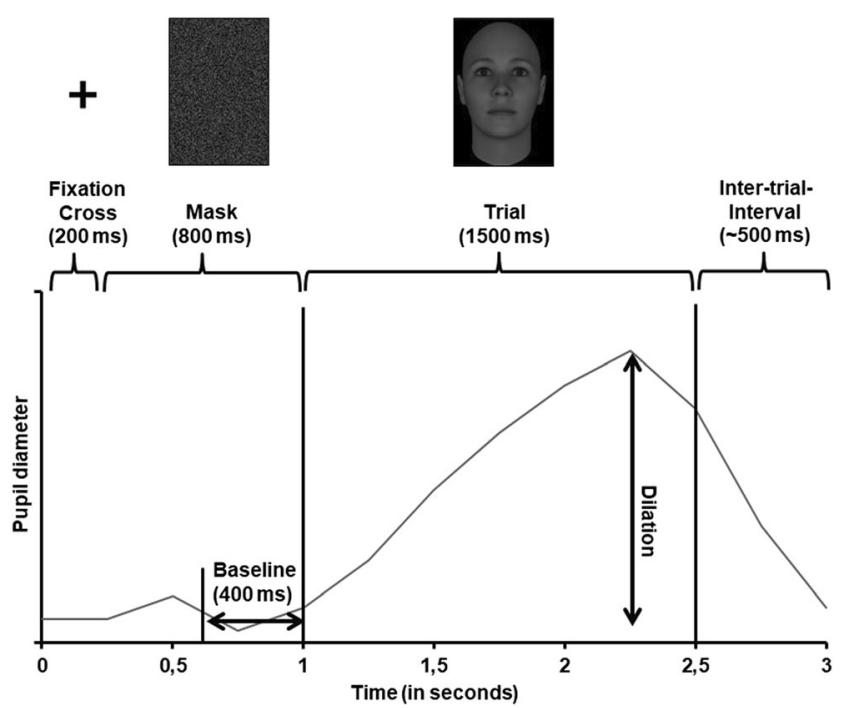

Fig. 1. Overview of the time course of one trial in the labor-leisure task, including the parts of the trial in which we measured baselines and dilations, respectively. Face stimulus taken from Said and Todorov (2011)

\section{Stimuli}

Faces were selected from the Attractiveness Model Database (Said \& Todorov, 2011), which contains artificially generated faces. Of the 20 faces used, 10 were drawn from the $5 \%$ most attractive faces of the database; the other 10 from the 5\% least attractive faces. Ten faces were male faces; the other 10 were female faces. All stimuli were matched on mean luminance and had the same size ( $400 \times 400$ pixels). The mask consisted of the pixels of one of the faces randomly rearranged, resulting in the same mean luminance. For all practice phases and the final test phase, we generated a pseudo-random order of faces, with a $30 \%$ chance of a 2-back trial occurring. Fifty percent of trials showed attractive faces.

\section{Control assessments}

In Study 2, after the labor-leisure task, participants additionally completed two control assessments of 80 trials $(5 \mathrm{~min})$ each. Both conditions were identical to the labor-leisure task in trial structure, stimuli, and task labels, but different in instructions.

First, in the motor control assessment, participants were instructed to respond to one particular face (and not to any other face) by moving the joystick in a way as if they switched between tasks in the labor-leisure task. This particular face was displayed on trials $5,15, \ldots 75$, with each of these trials surrounded by 10 trials without any action, allowing for a similar analysis of "switches" as in the labor-leisure task. With this basic vigilance task, we intended to test whether pupil changes were driven by the motor activity occurring when moving the joystick.
Second, in the visual control assessment, participants were instructed to passively watch a series of trials without any action. In trials $5,15, \ldots 75$, the frame highlighting the 2-back or attractiveness-rating task label was automatically shifted to the respective other task label. With this control assessment, we intended to test whether watching the moving frame was sufficient to induce the observed pupil changes.

\section{Questionnaires}

In both studies, after people had completed the tasks and left the eye-tracker, we administered three questionnaires:

The Irrational Procrastination Scale (IPS; Steel, 2010) measures general procrastination tendencies using nine items, such as "I often regret not getting to task sooner." People responded on a 7-point scale ranging from strongly disagree (1) to strongly agree (7).

The Action Control Scale (ACS-24; Kuhl, 1994) consists of 24 descriptions of scenarios, with 2 possible strategies to act in each situation. Participants are asked to select the strategy that describes best how they would react. It comprises two subscales: the action orientation subsequent to failure versus preoccupation (AOF) subscale contains scenarios about dealing with failures, such as "When several things go wrong on the same day," for which the action-oriented response is "I just keep on going as though nothing had happened" and the stateoriented one is "I don't know how to deal with it." In contrast, the prospective and decision-related action orientation versus hesitation (AOD) subscale contains items related to planning and starting activities, such as "When I have an obligation to do something that is boring and uninteresting" with the action-oriented response "I do it and get it over with" and the state-oriented response "It usually takes a while before I get around to doing it."

Furthermore, we selected three subscales from the SelfGovernment Inventory (SSI-K3; Kuhl \& Fuhrmann, 1998): the self-regulation (competence) subscale relates to feelings of autonomy, intrinsic motivation, and dealing with nervousness and contains items, such as "When my perseverance subsides, I know exactly how to motivate myself again." The self-control subscale comprises items about planning, prospection, and self-confidence, such as "If I have a lot to do, I work according to a plan (i.e., I have a schedule for my tasks)." The volitional development (action development) subscale consists of items reflecting initiative and readiness to act in contrast to postponement and procrastination, such as "If something has to be done, I begin doing it immediately." Participants rated how much each item applied to themselves on a four-point scale from "not at all" to "completely." For each of the scales, we calculated participants' average scores. 


\section{Data analysis}

Pre-processing of eye-tracking data Pre-processing of the pupil raw data included removing values of zero, removing abnormally fast pupil changes, deleting outliers, and finally imputing missing values using linear interpolation. Our exact analysis script was built on previous experimental work (Bijleveld, 2018), and it is available at https://osf.io/b9z4c/ (Study 1) and https://osf.io/ukgsh/ (Study 2).

Pupil measures For each trial, baseline pupil diameter and maximal pupil dilation were computed. Baseline pupil diameter was defined as the average pupil size during the last $400 \mathrm{~ms}$ of the mask presentation. Pupil dilation was defined as the difference between the maximal pupil size during face stimulus presentation and the baseline pupil diameter (for an illustration, see Fig. 1).

Generalized additive mixed models To account for autocorrelation in the pupil data, we followed recent suggestions (Baayen, Vasishth, Kliegl, \& Bates, 2017) to fit generalized additive mixed models (GAMMs) to time-series data using the mgcv package (version 1.8.22; Wood, 2017) in $\mathrm{R}$ (version 3.4.3; R Core Team, 2017). The unit of analysis were single trials. Trials were nested in bouts, with a bout formed by the five trials before and five trials after a switch. We used either pupil baseline or pupil dilation as the dependent variable, each maximum normalized per person. ${ }^{3}$ We used (a) trial number relative to switch and (b) the interaction between trial number and switch type (labor-to leisure vs. leisure-to-labor) as predictors. We fitted GAMMs with random slopes for trial number for each bout of each participant, which effectively reduced auto-correlation to levels $<0.15 .^{4}$ When testing for differences between switch types, we coded the predictor switch types as an ordered factor.

Note that GAMMs fit a smooth curve (consisting of thin plate regression splines) through all data points and test whether this curve is significantly different from a straight line (i.e., from zero) at any time point during the selected time window. Thus, GAMMs do not qualify the shape (e.g., linear

\footnotetext{
${ }^{3}$ Maximum-normalization was implemented by subtracting the minimum of an individual from all their values and dividing them by the person's maximum. This transformed each individual's values into the range from 0 to 1 , reducing the effect of outliers. All analyses were repeated with the raw pupil measures as well as with these measures z-standardized per person. In both cases, the same pattern of significant and non-significant results was obtained. ${ }^{4}$ As a control, we also fitted GAMMs with random smooths, featuring an ARIMA(1)-model based on the model with random slopes to account for auto-correlation. However, given that each bout comprised at most 10 (and often less) data points, fitting separate smooths for each bout restricted us to using 8 knots for each smooth only, further constraining the "wigglyness" of the underlying smooth we could possibly fit. Auto-correlations were hardly any further reduced. These models yielded $F$ - and $p$-values that were highly similar to those of the models with random slopes and led to the same conclusions.
}

vs. curvilinear) or direction (e.g., increase vs. decrease) of a change. Hence, we additionally fitted linear mixed-effects models to test for linear increases or decreases in pupil baseline and pupil dilation. In our interpretations, we gave priority to the results of the GAMMs as those (a) account for autocorrelations, and (b) our hypotheses were agnostic about how early and how long the predicted changes would occur.

Linear mixed-effects models We fitted linear mixed-effects models (LMEMs) using the lme4-package (version 1.1.15; Bates, Mächler, Bolker, \& Walker, 2015) with pupil baseline or pupil dilation as outcome variable and trial number relative to switch as sole predictor. When comparing the effects of switch types, we added the factor switch type and the interaction between trial number and switch type. Both outcome measures and relative trial numbers were standardized, so that regression coefficients can be interpreted as standardized regression weights. For the factor switch type, we employed sum-to-zero coding. Models contained a maximal random effects structure (Barr, Levy, Scheepers, \& Tily, 2013), with random intercepts and random slopes of trial number, switch type, and their interaction, both for each participant and for each bout of adjacent trials of each participant, and with all possible random correlations. We computed type-3-like $p$ values using $F$-tests with Kenward-Roger approximation for degrees of freedom (Singmann, Bolker, Westfall, \& Aust, 2018).

Power estimation We checked in a small pilot study $(N=7)$ whether participants followed the task instructions and switched between tasks often enough to yield sufficient power for testing our hypotheses with $N=30$. Participants switched on average 8.4 times from labor to leisure. Given $N=30$ and our initial hypothesis of analyzing the last ten but one trial before switches (see below), we expected to obtain 2,268 usable trials. In our pilot data, we obtained intra-class correlations of 0.67 for baselines and 0.37 for dilations. Following Aarts, Verhage, Veenvliet, Dolan, and van der Sluis (2014), we estimated effective sample sizes of 357 trials for baselines and 597 trials for dilations, which allowed us to detect effects of $\beta>0.14$ for baselines and $\beta>0.11$ for dilations with $80 \%$ power (power sensitivity analysis for linear bivariate regression in G*Power 3; Faul, Erdfelder, Lang, \& Buchner, 2007).

\section{Results}

\section{Manipulation checks}

Task ratings In Study 1, participants perceived the 2-back task ( $M=5.87, S D=0.85)$ as more work compared with the rating $\operatorname{task}(M=2.00, S D=1.03), t(30)=17.13, p<0.001, d=3.08$, but also as significantly less fun $(M=2.58, S D=0.92)$ 
compared with the rating task $(M=3.70, S D=1.53), t(30)=$ $-4.25, p<0.001, d=-0.76$. Also in Study 2, participants perceived the 2-back task as more work $(M=5.32, S D=$ $1.45)$ than the rating task $(M=2.32, S D=1.25), t(30)=$ $8.09, p<0.001, d=1.45$, while fun ratings did not significantly differ between the 2-back task $(M=2.84, S D=1.37)$ and the rating task $(M=2.94, S D=1.34), t(30)=0.31, p=$ $0.756, d=-0.06$. This resulted from a lower "fun" rating of the rating task compared with Study 1, which might have been due to the fact that in Study 2, between the test phase and the ratings, participants completed the control assessments for another 10 minutes. Hence, their memory of the tasks might have faded. Overall, we concluded that in both studies (a) the task successfully implemented labor in contrast to leisure, and (b) the task established a context in which not fun, but reward maximization, drove participants' choices about which task to perform.

Task performance In Study 1, on average, participants spent $396(S D=53)$ out of 500 trials on the labor task; they switched 7.97 times $(S D=7.64)$ from labor to leisure. In the labor task, participants gave on average $84 \%(S D=4 \%)$ correct responses. In the leisure task, they responded in $27 \%$ ( $S D=$ $14 \%$ ) of trials, indicating that participants actively engaged in this task. In Study 2, participants spent on average 352 trials $(S D=123)$ on the labor task; they switched 8.77 times $(S D=$ $6.58)$ from labor to leisure. In the labor task, they responded in $84 \%$ of trials $(S D=6 \%)$ correctly. During the leisure task, they responded in $28 \%(S D=17 \%)$ of trials. We conclude that in both studies, participants were engaged in both tasks, even though performance on the leisure task had no impact on their payout. See S3 for details on accuracy directly before switches to leisure, S4 for differences in accuracy before compared to after periods of leisure, and S5 for correlations of overall baseline pupil diameter and pupil dilations with performance measures.

Overall baseline and dilation differences between tasks To ensure that changes in pupil baselines or dilations around switches between tasks were not due to overall differences between tasks, we fitted LMEMs to the maximumnormalized baseline and dilation scores with task as the single predictor (including full random effects structures). In both studies, baselines (Study 1: $\mathrm{M}_{\text {labor }}=0.58, \mathrm{M}_{\text {leisure }}=0.54$, Study 2: $\mathrm{M}_{\text {labor }}=0.57, \mathrm{M}_{\text {leisure }}=0.56$ ) and dilations (Study $1: \mathrm{M}_{\text {labor }}=0.36, \mathrm{M}_{\text {leisure }}=0.35$; Study 2: $\mathrm{M}_{\text {labor }}=0.36, \mathrm{M}_{\text {leisure }}$ $=0.32$ ) tended to be higher during labor compared to leisure. However, these differences between tasks were only significant for dilations in Study 2, $F(1,27.51)=4.50, p=.043$ (all other $p \mathrm{~s}>0.11$ ), and never exceeded $\sim 0.04$ on a maximumnormalized scale. These differences are substantially smaller than baseline increases and dilation decreases around switches (see below) and thus cannot explain the observed pupil changes around switches. Furthermore, if there were no additional mechanisms driving pupil changes around switches, the finding of overall slightly higher baselines and dilations during the labor task would make us expect decreases in both measures around labor-to-leisure switches but increases in both measures around leisure-to-labor switches, which is inconsistent with our findings reported below.

\section{Pre-registered confirmatory analyses of Study 1}

In our original pre-registration, we decided to select the last ten but one trials before labor-to-leisure switches and fit LMEMs with the outcomes baseline pupil diameter or pupil dilations and the sole predictor trial number relative to the switch $(-10$ to -2$)$. There was no evidence for an increase in baseline pupil diameter, $\beta=0.01,95 \%$ CI $[-0.04,0.06], F(1$, $23.50)=0.11, p=0.743$, nor for a decrease in pupil dilations, $\beta=-0.03,95 \%$ CI $[-0.08,0.02], F(1,25.68)=1.33, p=$ 0.260 , during the pre-registered time window.

\section{Exploratory analyses Study 1 and pre-registered analyses Study 2}

As a next step, we ran exploratory analyses on the data from Study 1. For this purpose, we considered a different range of trials, namely the last five trials before and the first five trials after switches, centered on the trial on which participants decided to switch. This allowed us to investigate whether pupil changes started later than we initially expected, namely only one to two trials before the switch. Also, we investigated both switches from labor to leisure and from leisure to labor. Unless otherwise indicated, all analyses reported below were preregistered for Study 2.

Changes in baseline diameters Table 1 reports the results of the fitted GAMMs and LMEMs for both studies. GAMMs yielded significant changes in both baseline pupil diameter and pupil dilations, for both switch types, in both studies. Time courses of the GAMMs are plotted in Fig. 1. In Table 1, we further report LMEMs. We used these LMEMs to test whether there was a significant net increase or decrease (recognizable by the sign of the beta coefficient) across the entire specified trial window.

As predicted by both adaptive gain theory and network reset theory, GAMMs showed that baseline pupil diameter increased around labor-to-leisure switches in both Study 1 and Study 2 (Fig. 2a and b). Whereas LMEMs indicated that there also was an overall net increase in baselines in Study 1, there was no significant evidence for such an increase in Study 2. Nevertheless, Fig. 2b clearly displays an increase, which might be too temporally constrained to be picked up as a net increase by the LMEM. We thus ran an additional exploratory analysis by refitting this LMEM on a more restricted time 
Table 1 Results of generalized additive mixed models (GAMMs) and linear mixed-effects models (LMEMs) for pupil baseline diameter and pupil dilations across labor-to-leisure and leisure-to-labor switches in Study 1 and 2

\begin{tabular}{|c|c|c|c|c|}
\hline Measure & Switch type & Study & GAMM & LMEM \\
\hline \multirow[t]{6}{*}{ Baseline } & \multirow[t]{2}{*}{ Labor-to-leisure } & 1 & $F(8.54,3577.26)=23.65, p<0.001$ & \multirow{2}{*}{$\begin{array}{l}\beta=0.12,95 \% \text { CI }[0.04,0.19] \\
F(1,25.88)=9.89, p=0.004 \\
\beta=.03,95 \% \text { CI }[-0.02,0.08] \\
F(1,26.14)=1.56, p=0.222^{\mathrm{a}}\end{array}$} \\
\hline & & 2 & $F(8.30,4257.04)=11.43, p<0.001$ & \\
\hline & \multirow[t]{2}{*}{ Leisure-to-labor } & 1 & $F(8.50,3577.26)=23.95, p<0.001$ & \multirow{4}{*}{$\begin{array}{l}\beta=0.16,95 \% \mathrm{CI}[0.09,0.24], \\
F(1,27.85)=19.35, p<0.001 \\
\beta=0.12,95 \% \mathrm{CI}[0.07,0.17], \\
F(1,23.46)=23.46, p<0.001 \\
\beta=-0.02,95 \% \mathrm{CI}[-0.08,0.03], \\
F(1,26.56)=0.96, p=0.336 \\
\beta=0.04,95 \% \mathrm{CI}[-0.09,0.01], \\
F(1,26.72)=3.51, p=0.072\end{array}$} \\
\hline & & 2 & $F(8.57,4257.04)=21.46, p<0.001$ & \\
\hline & \multirow[t]{2}{*}{ Difference } & 1 & $F(1.00,3584.57)=1.16, p=0.281$ & \\
\hline & & 2 & $F(3.33,4261.67)=4.06, p=0.005$ & \\
\hline \multirow[t]{6}{*}{ Dilation } & \multirow[t]{2}{*}{ Labor-to-leisure } & 1 & $F(7.76,4012.88)=2.91, p=0.003$ & \multirow{6}{*}{$\begin{array}{l}\beta=-0.07,95 \% \text { CI }[-0.14,-0.01], \\
F(1,27.75)=4.90, p=0.035 \\
\beta=-0.06,95 \% \text { CI }[-0.02,0.11], \\
F(1,26.43)=6.71, p=0.015, \\
\beta=0.001,95 \% \text { CI }[-0.07,0.06], \\
F(1,28.17)=0, p=0.962^{\mathrm{a}} \\
\beta=0.01,95 \% \text { CI }[-0.02,0.05], \\
F(1,20.07)=0.51, p=0.482^{\mathrm{a}}, \\
\beta=-0.04,95 \% \text { CI }[-0.09,0.02], \\
\left.F(1,28.54)=1.95, p=0.174^{\mathrm{b}}\right] \\
\beta=-0.03,95 \% \text { CI }[-0.06,-0.01], \\
F(1,520.93)=7.79, p=0.005^{\mathrm{b}}\end{array}$} \\
\hline & & 2 & $F(1,4688.12)=13.31, p<0.001$ & \\
\hline & \multirow[t]{2}{*}{ Leisure-to-labor } & 1 & $F(7.91,4012.88)=5.13, p<0.001$ & \\
\hline & & 2 & $F(7.66,4688.12)=5.24 ; p<0.001$ & \\
\hline & \multirow[t]{2}{*}{ Difference } & 1 & $F(1.80,4002.19)=4.16, p=0.019$ & \\
\hline & & 2 & $F(7.76,4687.73)=6.29, p<0.001$ & \\
\hline
\end{tabular}

We fitted separate models for labor-to-leisure and leisure-to-labor switches testing our pre-registered hypotheses and then exploratory models to compare both switch types

${ }^{a}$ Estimates were significantly different from zero when we refitted them on a more constrained time window of two trials before until two trials after switches (see main text)

${ }^{\mathrm{b}}$ Due to convergence warnings, we simplified the model by dropping the random slopes either per bout or per subject until the model converged
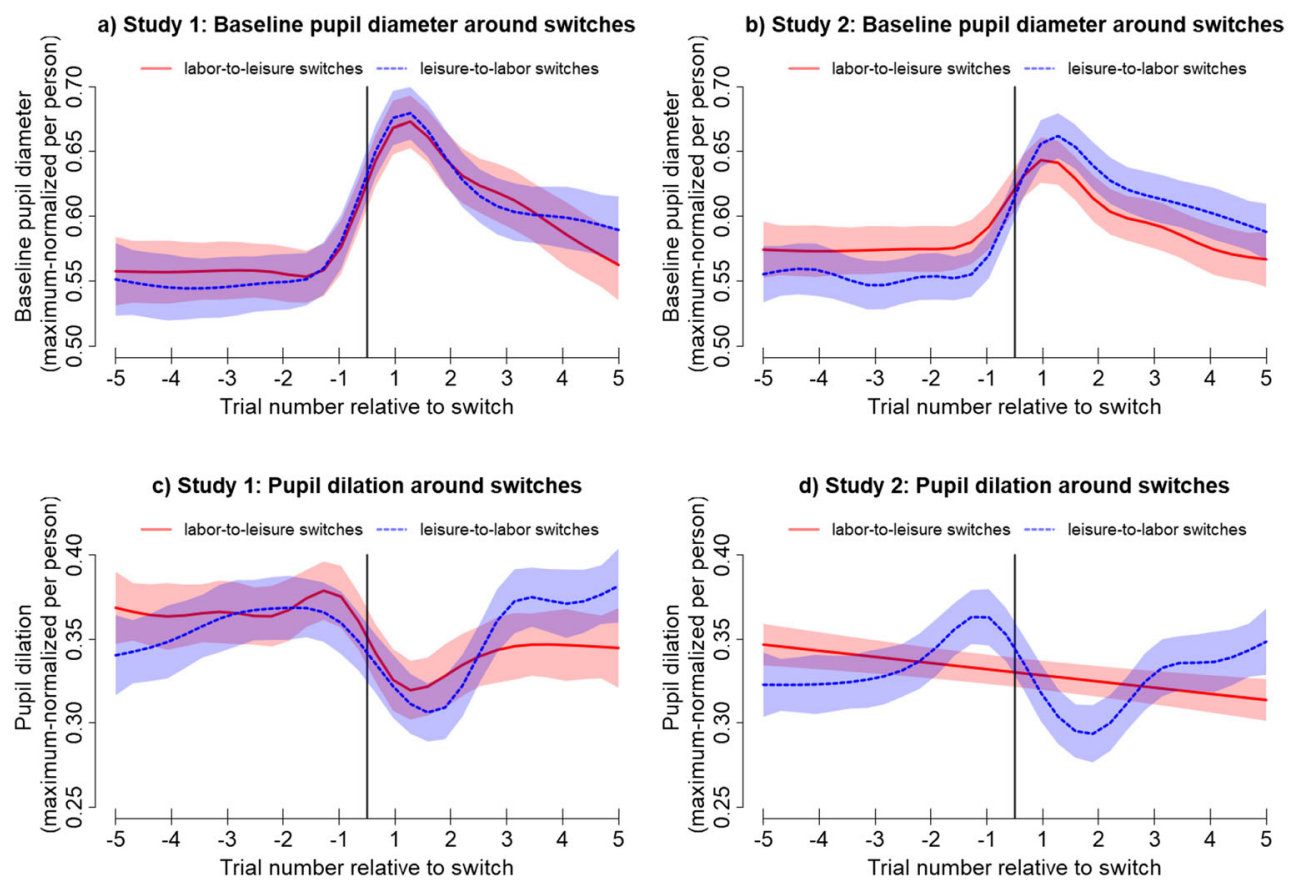

Fig. 2. Top panels: Time course of baseline pupil diameter around laborto-leisure and leisure-to-labor switches in Studies 1 (A) and 2 (B). Bottom panels: Time course of pupil dilations around labor-to-leisure and leisureto-labor switches in Studies 1 (C) and 2 (D). All time courses were

derived from the GAMMs described in Table 1 (time courses are model estimates, not averages of data). Shades indicate $95 \%$ CIs. Vertical lines indicate the time point of the switch 
window from two trials before until two trials after the switch, which yielded indeed a strong, but temporally constrained, increase, $\beta=0.13,95 \%$ CI $[0.07,0.20], F(1,27.30)=$ $13.19, p=0.001$.

Furthermore, in line with network reset theory, but in contradiction with adaptive gain theory, GAMMs yielded a similar increase in baselines across leisure-to-labor switches in both studies. This was corroborated by LMEMs in both studies, which both showed net increases in baselines across these switches.

We ran exploratory analyses to test whether the time courses of pupil dilations differed between switch types. When comparing switch types in Study 1, neither GAMMs nor LMEMs indicated evidence for a significant difference between switch types. In Study 2, the GAMM yielded that the increase in baselines was even slightly stronger for leisure-to-labor compared to labor-to-leisure switches (Fig. $2 \mathrm{~b}$ ), whereas the LMEM yielded no evidence for a net difference. Overall, in both studies, we found significant increases in pupil baselines in labor-to-leisure switches, which is consistent with both adaptive gain theory and network reset theory, as well as in leisure-to-labor switches, which is consistent with network reset theory, but inconsistent with adaptive gain theory.

Changes in pupil dilations For labor-to-leisure switches, as predicted by both adaptive gain theory and network reset theory, GAMMs revealed significant decreases in pupil dilations in both studies (Fig. 2c and d). This was corroborated by LMEMs indicating significant net decreases in dilations in both studies.

For leisure-to-labor switches, GAMMs indicated a similar decrease in both studies, which was in line with network reset theory, but inconsistent with adaptive gain theory. However, LMEMs did not yield evidence for a significant net decrease in either study. Given that decreases were clearly visible in Fig. 2c and d, we again ran exploratory analyses by refitting the models on a more temporally restricted trial window from two trials before until two trials after a switch. In this window, there was a strong significant decrease both in Study $1, \beta=$ $-0.16,95 \%$ CI $[-0.24,-0.08], F(1,21.94)=15.36, p<0.001$, and Study $2, \beta=-0.13,95 \% \mathrm{CI}[-0.19,-0.07], F(1,21.07)=$ $17.41, p<0.001$.

We ran exploratory analyses to test whether the time courses of pupil dilations differed between switch types. As shown in Fig. 2c, GAMMs indicated that dilation time courses were significantly different between switch types in Study 1, with dilations higher three to five trials after leisure-to-labor switches compared with labor-to-leisure switches. The respective LMEM yielded no evidence for net differences. In Study 2, both GAMMs and LMEMs indicated significant differences between switch types. Figures $2 \mathrm{~d}$ shows that dilations were higher five trials before and two trials after labor-to- leisure compared with leisure-to-labor switches but lower one trial before and five trials after labor-to-leisure compared with leisure-to-labor switches.

We make two more observations about the particular shape of the pupil dilation time courses across switches. First, note that in in both studies, before the decrease, there seems to be a brief increase on the last trial before a switch (Fig. 2c and d). Although neither adaptive gain theory nor network reset theory predicts this increase, it may be explained by the idea that pupil dilation reflects effort mobilization (Beatty \& LuceroWagoner, 2000; Hess \& Polt, 1964; Kahneman \& Beatty, 1966; Laeng, Sirois, \& Gredebäck, 2012). It may be the case that initiating the task switch requires particular effort (see also the motor control assessments below).

Second, regarding labor-to-leisure switches, the GAMM for Study 1 displays a somewhat abrupt decrease that is constrained to one to two trials around switches (Fig. 2c, red line), while the GAMM for Study 2 (Fig. 2d, red line) displays a more gradual decrease over the entire trial window. Again, neither adaptive gain theory nor network reset theory make predictions about the exact shape of the decrease. However, the difference between studies may be explained from the way GAMM models fit smooth curves through data. In GAMM models, the order of the best-fitting polynomial is determined by trading off model fit versus model complexity. For a noisier raw data pattern (with multiple peaks and troughs), this tradeoff may favor a lower order polynomial (as in Fig. 2d), which is more likely to reflect the data generative process. When we visually inspected pupil dilation time courses (for the script generating the respective plots, see https://osf.io/ukgsh/), we did indeed observe that the dilation data for labor-to-leisure switches seemed to be noisier in Study 2 than Study 1, which may explain why the decrease in pupil dilation seemed to be more gradual in Study 2.

Pupil changes around switches compared with overall differences between tasks A possible explanation for changes in pupil baselines and dilations around task switches is that those measures are overall higher in one task compared with the other. If this was the case, pupil changes around switches would merely reflect the transition from one task to the other, rather than particular processes that are involved in implementing the task switch. To rule out this possibility, we compared the magnitude of pupil changes around switches (Fig. 2) to overall differences between tasks on those measures. Baseline increases around switches were of magnitudes $\sim 0.15$ on the maximum-normalized scale and dilation decreases of magnitudes $\sim 0.10$, which was both substantially larger than overall differences between tasks on those measures (which did not exceed $\sim 0.04$ ). We thus deem it unlikely that the observed baseline and dilation changes around switches are reducible to overall differences between tasks on those pupil measures. 
Summary of main results In conclusion, across both studies, we found increases in pupil baseline levels and decreases in pupil dilations around both labor-to-leisure and leisure-tolabor task switches. The size of baseline increases and dilation decreases was largely comparable between both switch types and across both studies.

These results were fully predicted by network reset theory. Only the patterns observed for labor-to-leisure switches, but not the pattern for leisure-to-labor switches, was consistent with adaptive gain theory. In sum, our data provided support for network reset theory, which proposes that pupil shifts across both types of task switches reflect processes involved in task-switching, independent of the motivational nature of the task. However, one might argue that these shifts are comparable across switches types and studies, because they do not reflect neural processes underlying task switching, but rather (a) motor processes initiating the movement of the joystick, or (b) visual processes of observing the frame highlighting the current task moving from one task label to the other. Although these alternative explanations seem rather unlikely given the prolonged elevation of pupil baselines until 12 seconds after the behavioral switch, we aimed to rule out those alternative explanations via two additional assessments.

\section{Control assessments (Study 2 only)}

\section{Motor control assessment}

A GAMM indicated that contrary to our expectations, the time course of baseline pupil diameter around motor actions was significantly different from zero, $F(8.93,1535.64)=40.40, p$ $<0.001$ (Figure S6a), and a LMEM yielded a significant increase, $\beta=0.11,95 \%$ CI $[0.05,0.16], F(1,22.00)=15.25, p<$ 0.001 . Additionally, contrary to our expectations, a GAMM indicated that the time course of pupil dilation was significant different from zero, $F(8.94,1665.19)=20.47, p<0.001$ (Figure S6c), with a LMEM yielding a significant decrease, $\beta=-0.08,95 \%$ CI $[-0.12,-0.03], F(1,21.99)=10.63, p=$ 0.004 .

Several factors might account for this unexpected finding. First, we expected this condition to be an easy task, but participants committed a considerable amount of errors (217 of 2,480 trials, i.e., $10 \%$ of all trials; with 148 false-positive switches and 69 false-negative nonswitches). ${ }^{5}$ This indicates that the task was more difficult (or less engaging) than expected, and effortful processes different from mere motor activation (e.g., working memory) might have contributed to pupil changes (Kahneman, 1973). Second, it seemed that pupil dilations were particularly high in the motor control assessments on the very last trial before a switch, i.e., the trial participants

\footnotetext{
$\overline{5}$ For appropriate exclusion of participants and trials from the following analyses, see SOM S2.
}

saw the face that triggered them to switch, which could reflect increased effort (Hess \& Polt, 1964; Kahneman \& Beatty, 1966). In an exploratory analysis, we directly contrasted the time courses in the motion control assessment with those around leisure-to-labor switches, using a GAMM with the interaction between trial number and the ordered factor switch type (motor control assessment vs. leisure-to-labor). ${ }^{6}$ The differences in the time course of pupil dilation was indeed significant, $F(8.93,3975.57)=12.88, p<0.001$, indicating that pupil dilations were higher in the motor control assessment on the very last trial before a switch, but higher in leisure-to-labor switches on the very first trial after a switch (Figure S6d). Third, it seemed that baseline increases in motor control assessments were restricted to the very first trial after a switch but more sustained in labor-to-leisure and leisure-to-labor switches. When directly contrasting the time courses of pupil baselines of motor control assessments and leisure-to-labor switches, we indeed found significant differences, $F(8.57$, $3657.29)=12.27, p<0.001$ : baselines were higher in leisure-to-labor switches from five to one trial before the switch and from two to five trials after the switch, but the motor control assessment was only higher on the very first trial after a switch (Figure S6b).

The motor control assessment might have been more difficult for participants than we expected, as indicated by the high error rates and the strong pupil dilation on the last trial before the switch, which might reflect increased effort recruitment. Also, increases in the labor-to-leisure and leisure-to-labor switches continued for several trials after a switch, while the increase in the motor control assessment was only present on the very first trial after a switch. These findings render it unlikely that the pupil changes observed in labor-to-leisure and leisure-to-labor switches were fully reducible to processes underlying motor movements.

\section{Visual control assessment}

In the selected time window, contrary to our expectations, a GAMM indicated that the time course of baselines in the visual control assessment was significantly different from zero, $F(6.63,2075.75)=2.92, p=0.006$. Visual inspection showed however that the time course was overall rather flat (Figure S6a). ${ }^{7}$ A LMEM found no evidence for any net changes across the selected time window, $\beta=-0.04,95 \% \mathrm{CI}$ $[-0.09,0.01], F(1,30.00)=3.63, p=0.067$. Similarly, a

\footnotetext{
${ }^{6}$ Pupil changes around leisure-to-labor switches were overall larger than those around labor-to-leisure switches and thus should be more similar to the time course in the motor control assessment, providing a more conservative test for differences. The same results were found when comparing switches in the motor control assessment to labor-to-leisure switches.

${ }^{7}$ When fitted with random smooths on eight knots, the time course in the visual control assessment was not significantly different from a flat line, $\mathrm{F}(2.88,1695.85)=2.21, p=0.060$.
} 
GAMM found no evidence for the time course of pupil dilations deviating from a flat line, $F(2.48,2335.32)=2.09, p=$ 0.112 (Figure S6b), and a LMEM found no evidence for net changes either, $\beta=0.01,95 \% \mathrm{CI}[-0.03,0.04], F(1,30.00)=$ $0.04, p=0.835$. We conclude that the pupil changes observed in labor-to-leisure and leisure-to-labor switches do not stem from merely watching the frame highlighting task labels move.

\section{Correlations with action orientation across both studies}

We initially pre-registered to run LMEMs with the predictors trial number, switch type, the respective questionnaire scale, and all interactions to check whether individual differences in procrastination and action orientation predicted the amplitude of pupil changes, and differently so for the different switch types. Because these models failed to converge, we instead reused the simple models of baseline increases and dilation decreases reported above, extracted the best linear unbiased predictors (BLUPs), i.e., the group-level fixed effect slope of trial number plus the respective random slope of trial number of each participant, and correlated those with participants' average scores on the questionnaires. ${ }^{8}$ Note that we did not compare correlations against zero, but against the correlations of the respective other switch type. Correlations between BLUPs and questionnaires are displayed in Table 2; descriptive statistics and intercorrelations between questionnaires in SOM S7. Note that pupil dilation decreases around switches, so BLUPs are typically negative; the more negative they are, the larger is the amplitude of the decrease. Most correlations were low. As the most noteworthy finding, which we think might warrant follow-up research, we observed that the BLUPs of pupil dilation decreases were positively associated with action orientation after failure (AOF) and self-regulation (SR) for labor-to-leisure switches, but negatively for leisure-to-labor switches. The same pattern, although weaker, was found for the subscales decision-related action orientation (AOD) and self-control (SC). ${ }^{9}$ The negative correlations with BLUPs in leisure-to-labor switches imply that the higher participants scored on action orientation, the stronger were their decreases in pupil dilation in leisure-to-labor switches, but the weaker were their decreases in pupil dilation in labor-to-leisure switches. However, given our limited sample size of only 62 participants, those findings might not be robust and need further corroboration by future research. No associations were found with procrastination (IPS) and volitional development (VD).

\footnotetext{
${ }_{8}^{8}$ BLUPs were fitted based on the total time window of interest, even though on a group-level, there was no significant decrease in dilations across leisureto-labor switches in either study. This does not exclude the existence of meaningful individual differences between participants.

${ }_{9}$ All of these patterns also were reflected in significant or marginally significant 3-way interactions in the initially pre-registered LMEMs, which however yielded convergence warnings.
}

\section{Discussion}

In exploratory analyses (Study 1) and confirmatory analyses (Study 2), we found that pupil baseline levels increased and pupil dilations decreased around switches from cognitive labor to cognitive leisure, and from leisure to labor. These changes in pupil baseline levels and pupil dilations were short-lived. Our findings extend previous studies that found similar pupil changes when people disengaged from (and restarted) an auditory discrimination task (Gilzenrat et al., 2010), when people shifted between multiple bandit gambling machines (Jepma \& Nieuwenhuis, 2011) and when people shifted between strategies in solving Raven's Matrices (Hayes \& Petrov, 2016). By contrast to these previous studies, in our research, participants chose between two motivationally different tasks - namely an effortful, but profitable 2-back (labor) and an effortless, but unprofitable attractiveness rating task (leisure). Participants showed the same pupillary changes around switches between tasks in either direction. We will now discuss the main findings in greater detail.

\section{Pupil changes were similar for labor-to-leisure and leisure-to-labor switches}

Similar pupil changes (increased baseline, decreased dilations) occurred around switches from labor to leisure and in switches from leisure back to labor. While the former finding (labor to leisure) is consistent with adaptive gain theory, the latter finding (leisure to labor) is not. Thus, it seems that pupillary changes around task switches cannot be interpreted as reflecting people's motivation to take a break. Rather, these pupillary changes might reflect cognitive processes that underlie task switches more generally. This interpretation is in line with some predictions from network reset theory, which suggests that increases in pupil size (putatively reflecting NE release) occur when reorientation towards a new environment is needed. This reorientation account fits previous research that found increases in pupil diameter in response to environmental instabilities and surprises (Lavín, San Martín, \& Rosales Jubal, 2014; Nassar et al., 2012; Preuschoff, t' Hart, \& Einhäuser, 2011) and in response to rule changes in the Wisconsin Card Sorting Task (Pajkossy, Szőllősi, Demeter, \& Racsmány, 2017). Indeed, recent research found increased BOLD signals in the LC during task switching in humans (von der Gablentz, Tempelmann, Münte, \& Heldmann, 2015).

Thus, at least on first sight, our findings seem more consistent with predictions from network reset theory than with predictions from adaptive gain theory. We hasten to add, however, that it is debatable whether our data are in line with all aspects of network reset theory. In particular, network reset theory is typically used to understand how unexpected changes in environmental requirements drive NE-related reorientation of cortical networks (and its downstream behavioral consequences). It is not yet clear to what 
Table 2 Correlations of self-reported procrastination tendencies and action vs. state orientation with the Best Linear Unbiased Predictors (BLUPs) of pupil baseline diameter increases and pupil dilation decreases in both labor-to-leisure and leisure-to-labor switches

\begin{tabular}{llllll}
\hline & \multicolumn{2}{l}{ BLUPs baseline } & & \multicolumn{2}{l}{ BLUPs dilation } \\
\cline { 2 - 3 } \cline { 5 - 6 } & Labor-to-leisure switches & Leisure-to-labor switches & & Labor-to-leisure switches & Leisure-to-labor switches \\
\hline IPS & -0.04 & 0.01 & -0.09 & 0.14 & 0.01 \\
ACS-24 AOF & 0.17 & 0.05 & 0.19 & -0.28 \\
ACS-24 AOD & 0.14 & -0.11 & 0.07 & -0.13 \\
SSI K3 SR & 0.07 & 0.20 & 0.26 & -0.27 \\
SSI K3 SC & -0.04 & -0.14 & 0.16 & -0.25 \\
SSI K3 VD & -0.01 & 0.10 & 0.03 \\
\hline
\end{tabular}

Higher IPS values indicate higher procrastination tendencies, and higher values on the other scales indicate higher action orientation. Samples of both studies (total $N=62$ ) were combined. IPS = Irrational Procrastination Scale; ACS-24 AOF = ACS-24 Action orientation subsequent to failure vs. preoccupation subscale; ACS-24 AOD = ACS-24 Prospective and decision-related action orientation vs. hesitation subscale; SSI-K3 SR = SSI-K3 Selfregulation (Competence) subscale; SSI-K3 SC = SSI-K3 Self-control subscale; SSI-K3 VD = SSI-K3 Volitional development (Action development) subscale

$\dagger p<0.10, * p<0.05$, uncorrected

extent network reset theory can help to model the effects of people's self-initiated decisions, too.

Nevertheless, research does lean towards the idea that network reset theory applies to self-initiated decisions. In particular, one author of network reset theory (Sara, 2015, 2016) recently cited evidence for LC involvement in a task switching paradigm in humans (von der Gablentz et al., 2015) as evidence for network reset theory. In this paradigm, participants were not explicitly informed about task switching requirements. Instead, they had to infer when they needed to switch from performance feedback and from their own confidence in having responded correctly. Thus, it appears that also task switches that do not follow from evident changes in the environment, but from an inference process, may activate the LC. Using the same rationale, our results might extend network reset theory by suggesting that $\mathrm{NE}$ responses to the detection of environmental changes, such as threats or dangers, also might occur as responses to people's self-initiated decision to switch between tasks, perhaps helping them to adapt to the new task's requirements.

Related to the latter line of reasoning, some recent studies have suggested that NE plays a more active role in updating action policies (O'Reilly et al., 2013; Urai, Braun, \& Donner, 2017; Van Slooten, Jahfari, Knapen, \& Theeuwes, 2018), helping people to adopt new task mindsets, rather than (or in addition to) the role of encoding environmental uncertainty (Dayan \& Yu, 2006; Lavín et al., 2014; Nassar et al., 2012). Such a proposed role for NE in reconsidering action policies is consistent with recent electrophysiological work in monkeys that showed that (a) the activity of dopaminergic neurons in the substantia nigra correlates with motivational variables, such as expected levels of reward and effort, potentially integrating different signals into one single decision, but (b) activity of NE neurons in the LC correlates with actual effort exertion, potentially aiding the execution of this decision (Varazzani, San-Galli, Gilardeau, \& Bouret, 2015).

In sum, building on our findings and on recent insights in NE function, it is possible that previous experiments about shifts from exploitation to exploration (Gilzenrat et al., 2010; Hayes \& Petrov, 2016; Jepma \& Nieuwenhuis, 2011; Kane et al., 2017; Pajkossy et al., 2017) observed NE facilitating the execution of task switching, rather than NE facilitating motivational shifts. By contrast to these previous studies, our paradigm used two qualitatively distinct tasks, with distinct motivational properties (i.e., labor vs. leisure). Due to this design feature, our study could reveal that the motivational direction does not matter.

\section{Pupil changes were short-lived}

Our studies provide a fine-grained examination of the time course of baseline pupil diameter and pupil dilations around task switches. For both switches from labor to leisure and vice versa, changes in baselines and dilations began later than initially expected. In particular, baselines started to increase on the last trial ( 3 seconds) before a switch and levelled off around four trials (12 seconds) after the switch (Fig. 2a and b). Generally, dilations decreased from one trial before ( $3 \mathrm{sec}-$ onds) until two trials after a switch (6 seconds) and then returned to preswitch levels (exception: labor-to-leisure switches did not show this effect in Study 2, potentially due to measurement noise). Overall, changes in baselines and dilations were comparable between both switch types and across both studies. Importantly, both baselines and dilations returned to preswitch levels after a few trials, which was reflected in that our linear mixed-effect models did not always yielded a net change over the entire trial window ( 30 seconds).

The short-livedness of pupil changes is potentially relevant to our evaluation of adaptive gain theory and network reset theory. 
In particular, adaptive gain theory predicts that for extended periods of exploration, pupil baselines are permanently high and dilations permanently low. Such extended periods of exploration may take the form of mind wandering, during which people may plan for future activities (Kool \& Botvinick, 2014). In line with this idea, some studies found increased pupil baselines during mind-wandering (Franklin, Broadway, Mrazek, Smallwood, \& Schooler, 2013), whereas others found lower baselines (Grandchamp, Braboszcz, \& Delorme, 2014; Konishi, Brown, Battaglini, \& Smallwood, 2017; Mittner et al., 2014; Unsworth $\&$ Robison, 2016, 2018). Network reset theory, by contrast, predicts rapid adaptations around task switches. In this regard, our data seem to be consistent with predictions derived from network reset theory, as well.

\section{Discoveries from exploratory analyses}

Exploratory analyses suggested that individual differences in pupil dilation changes around switches were correlated with individual differences in self-reported action orientation. In particular, participants with higher action orientation showed smaller decreases in pupil dilation around switches from labor to leisure, but larger decreases in pupil dilation around switches from leisure to labor. This association might be plausible when the neural processes reflected by pupil dilation decreases help humans inhibit an old task mindset and prepare a new one, as proposed by network reset theory. In this case, inhibition and preparation are especially needed when going back to labor, because labor is both effortful and returns rewards, but not when switching to leisure. One might speculate that people who more selectively use their reorientation mechanisms in situations in which they are actually needed, i.e., when preparing an effortful task, also may approach challenges more proactively in everyday life.

In line with the latter line of reasoning, previous research indeed found action-oriented individuals to use their working memory capacity more effectively (Jostmann \& Koole, 2007) than state-oriented individuals. A similar association was recently discussed for fluid intelligence (Hayes \& Petrov, 2016), suggesting that more intelligent individuals show stronger pupil dilations compared to average-intelligent individuals in challenging tasks (van der Meer et al., 2010), but weaker dilations in simple tasks (Ahern \& Beatty, 1979). However, given that we did not predict this association a-priori, these results should be considered exploratory, and our interpretation should be considered speculative, requiring further corroboration by future work. It is worth noting that no such correlations were found for differences in baseline increases. The statistical power for these correlational analyses was quite low in our study. Thus, future research is needed to clarify the role of individual differences, with individual differences in action orientation as a potential starting point.

\section{Conclusions and future directions}

Our results showed that the pupil dynamics that often are thought to reflect shifts from exploitation to exploration also occur in shifts from exploration back to exploitation. Our results also indicated that these pupillary changes were shortlived; they disappeared within seconds. Although with caution, we suggest that pupillary shifts around task switches may not reflect a motivational process (people wanting to take a break) but instead a reorientation process (people preparing for the new task).

A limitation of our approach is that we used research on the exploration-exploitation trade-off to generate hypotheses about the labor-leisure trade-off. As noted in the Introduction, those two trade-offs might co-occur under particular circumstances but are prima facie independent. While the mapping of our tasks on the categories labor and leisure is likely stable, the mapping on exploitation and exploration might vary over time, e.g., when during the leisure task, participants suddenly detect the chance to indulge in a more abstract, intrinsic reward, such as a pleasant memory of an event outside the task context. Thus, more theoretical work-potentially using computational modeling to estimate the dynamic values of different goals, including extrinsic, monetary goals and intrinsic, more hedonic goals - is needed to draw conclusions about when a certain activity constitutes an exploitative option and when not (Meyniel et al., 2013; Mittner et al., 2014).

Future research might more directly test whether changes in pupil baselines and dilations are independent from the motivational properties of tasks. Also, future research might include control experiments that test whether the same pupil changes occur when task switches are externally induced (e.g., when participants see a countdown that requires them to switch). Such a study could elucidate whether externally induced switches (as classically concerned by network reset theory) and self-directed switches as implemented in our task share common mechanisms, e.g., the updating of action policies. Finally, it might be interesting to investigate further whether the magnitude of pupil changes is moderated by the difficulty of an upcoming task or by individual differences in action orientation.

Acknowledgments The authors thank H. Voogd for assistance with programming the eye-tracker and J. Kuhl for his kind permission to use the questionnaires that he created. J.A. thanks the Behavioural Science Institute at Radboud University for funding the research of his Master's thesis. E.B. was supported by grant 016-165-100 from the Netherlands Organization for Scientific Research.

Data and code availability Materials used in data collection as well as data and code to reproduce our analyses are available under https://osf.io/ b9z4c/ (Study 1) and https://osf.io/ukgsh/ (Study 2). 
Open Access This article is distributed under the terms of the Creative Commons Attribution 4.0 International License (http:// creativecommons.org/licenses/by/4.0/), which permits unrestricted use, distribution, and reproduction in any medium, provided you give appropriate credit to the original author(s) and the source, provide a link to the Creative Commons license, and indicate if changes were made.

\section{References}

Aarts, E., Verhage, M., Veenvliet, J. V, Dolan, C. V, \& van der Sluis, S. (2014). A solution to dependency: Using multilevel analysis to accommodate nested data. Nature Neuroscience, 17(4), 491-496. https://doi.org/10.1038/nn.3648

Ahern, S., \& Beatty, J. (1979). Pupillary responses during information processing vary with Scholastic Aptitude Test scores. Science, 205(4412), 1289-1292. https://doi.org/10.1126/science.472746

Aston-Jones, G., \& Cohen, J. D. (2005). An integrative theory of locus coeruleus-norepinephrine function: Adaptive gain and optimal performance. Annual Review of Neuroscience, 28, 403-450. https://doi. org/10.1146/annurev.neuro.28.061604.135709

Baayen, H., Vasishth, S., Kliegl, R., \& Bates, D. (2017). The cave of shadows: Addressing the human factor with generalized additive mixed models. Journal of Memory and Language, 94(5), 206234. https://doi.org/10.1016/j.jml.2016.11.006

Barr, D. J., Levy, R., Scheepers, C., \& Tily, H. J. (2013). Random effects structure for confirmatory hypothesis testing: Keep it maximal. Journal of Memory and Language, 68(3), 255-278. https://doi. org/10.1016/j.jml.2012.11.001

Bates, D., Mächler, M., Bolker, B., \& Walker, S. (2015). Fitting linear mixed-effects models using lme4. Journal of Statistical Software, 67(1), 1-48. https://doi.org/10.18637/jss.v067.i01

Beatty, J., \& Lucero-Wagoner, B. (2000). The pupillary system. In J. T. Cacioppo, L. G. Tassinary, \& G. Berntson (Eds.), Handbook of psychophysiology (2nd ed., pp. 142-162). Cambridge, MA: Cambridge University Press.

Berridge, C. W., \& Waterhouse, B. D. (2003). The locus coeruleusnoradrenergic system: Modulation of behavioral state and statedependent cognitive processes. Brain Research Reviews, 42(1), 33-84. https://doi.org/10.1016/S0165-0173(03)00143-7

Bijleveld, E. (2018). The feeling of effort during mental activity. Consciousness and Cognition, 63(February), 218-227. https://doi. org/10.1016/j.concog.2018.05.013

Bijleveld, E., Custers, R., \& Aarts, H. (2009). The unconscious eye opener: Pupil dilation reveals strategic recruitment of resources upon presentation of subliminal reward cues. Psychological Science, 20(11), 1313-1315. https://doi.org/10.1111/j.1467-9280.2009. 02443.x

Bijleveld, E., Custers, R., \& Aarts, H. (2012). Adaptive reward pursuit: How effort requirements affect unconscious reward responses and conscious reward decisions. Journal of Experimental Psychology: General, 141(4), 728-742. https://doi.org/10.1037/a0027615

Bouret, S., \& Sara, S. J. (2005). Network reset: A simplified overarching theory of locus coeruleus noradrenaline function. Trends in Neurosciences, 28(11), 574-582. https://doi.org/10.1016/j.tins. 2005.09.002

Calhoun, A. J., \& Hayden, B. Y. (2015). The foraging brain. Current Opinion in Behavioral Sciences, 5, 24-31. https://doi.org/10.1016/ j.cobeha.2015.07.003

Charnov, E. L. (1976). Optimal foraging, the marginal value theorem. Theoretical Population Biology, 9(2), 129-136. https://doi.org/10. 1016/0040-5809(76)90040-X

Cohen, J. D., McClure, S. M., \& Yu, A. J. (2007). Should I stay or should I go? How the human brain manages the trade-off between exploitation and exploration. Philosophical Transactions of the Royal
Society, B: Biological Sciences, 362(1481), 933-942. https://oi. org/10.1098/rstb.2007.2098

Constantino, S. M., \& Daw, N. D. (2015). Learning the opportunity cost of time in a patch-foraging task. Cognitive, Affective, \& Behavioral Neuroscience, 15(4), 837-853. https://doi.org/10.3758/s13415-0150350-y

Dayan, P., \& Yu, A. J. (2006). Phasic norepinephrine: A neural interrupt signal for unexpected events. Network: Computation in Neural Systems, 17(4), 335-350. https://doi.org/10.1080/ 09548980601004024

Faul, F., Erdfelder, E., Lang, A.-G., \& Buchner, A. (2007). G*Power 3: A flexible statistical power analysis program for the social, behavioral, and biomedical sciences. Behavior Research Methods, 39(2), 175191. https://doi.org/10.3758/BF03193146

Franklin, M. S., Broadway, J. M., Mrazek, M. D., Smallwood, J., \& Schooler, J. W. (2013). Window to the wandering mind: Pupillometry of spontaneous thought while reading. Quarterly Journal of Experimental Psychology, 66(12), 2289-2294. https:// doi.org/10.1080/17470218.2013.858170

Gilzenrat, M. S., Nieuwenhuis, S., Jepma, M., \& Cohen, J. D. (2010). Pupil diameter tracks changes in control state predicted by the adaptive gain theory of locus coeruleus function. Cognitive, Affective, \& Behavioral Neuroscience 10(2), 252-269. https://doi.org/10.3758/ CABN.10.2.252

Grandchamp, R., Braboszcz, C., \& Delorme, A. (2014). Oculometric variations during mind wandering. Frontiers in Psychology, 5(FEB), 1-10. https://doi.org/10.3389/fpsyg.2014.00031

Hayden, B. Y. (2018). Economic choice: The foraging perspective. Current Opinion in Behavioral Sciences, 24, 1-6. https://doi.org/ 10.1016/j.cobeha.2017.12.002

Hayes, T. R., \& Petrov, A. A. (2016). Pupil diameter tracks the exploration-exploitation trade-off during analogical reasoning and explains individual differences in fluid intelligence. Journal of Cognitive Neuroscience, 28(2), 308-318. https://doi.org/10.1162/ jocn_a_00895

Hess, E. H., \& Polt, J. M. (1964). Pupil size in relation to mental activity during simple problem-solving. Science, 143(3611), 1190-1192. https://doi.org/10.1126/science.143.3611.1190

Hills, T. T., Todd, P. M., Lazer, D., Redish, A. D., \& Couzin, I. D. (2015). Exploration versus exploitation in space, mind, and society. Trends in Cognitive Sciences, 19(1), 46-54. https://doi.org/10.1016/j.tics. 2014.10.004

Hunt, L. T., \& Hayden, B. Y. (2017). A distributed, hierarchical and recurrent framework for reward-based choice. Nature Reviews Neuroscience, 18(3), 172-182. https://doi.org/10.1038/nrn.2017.7

Inzlicht, M., Schmeichel, B. J., \& Macrae, C. N. (2014). Why self-control seems (but may not be) limited. Trends in Cognitive Sciences, 18(3), 127-133. https://doi.org/10.1016/j.tics.2013.12.009

Jepma, M., \& Nieuwenhuis, S. (2011). Pupil diameter predicts changes in the exploration-exploitation trade-off: Evidence for the Adaptive Gain Theory. Journal of Cognitive Neuroscience, 23(7), 15871596. https://doi.org/10.1162/jocn.2010.21548

Jett, Q. R., \& George, J. M. (2003). Work interrupted: A closer look at the role of interruptions in organizational life. Academy of Management Review, 28(3), 494-507.

Joshi, S., Li, Y., Kalwani, R. M., \& Gold, J. I. (2016). Relationships between pupil diameter and neuronal activity in the locus coeruleus, colliculi, and cingulate cortex. Neuron, 89(1), 221-234. https://doi. org/10.1016/j.neuron.2015.11.028

Jostmann, N. B., \& Koole, S. L. (2007). On the regulation of cognitive control: Action orientation moderates the impact of high demands in Stroop interference tasks. Journal of Experimental Psychology: General, 136(4), 593-609. https://doi.org/10.1037/0096-3445.136. 4.593

Kahneman, D. (1973). Attention and effort. Englewood Cliffs, NJ: Prentice Hall. 
Kahneman, D., \& Beatty, J. (1966). Pupil diameter and load on memory. Science, 154(3756), 1583-1585. https://doi.org/10.1126/science. 154.3756 .1583

Kane, G. A., Vazey, E. M., Wilson, R. C., Shenhav, A., Daw, N. D., Aston-Jones, G., \& Cohen, J. D. (2017). Increased locus coeruleus tonic activity causes disengagement from a patch-foraging task. Cognitive, Affective, \& Behavioral Neuroscience https://doi.org/10. 3758/s13415-017-0531-y

Kolling, N., Behrens, T. E. J., Mars, R. B., \& Rushworth, M. F. S. (2012). Neural mechanisms of foraging. Science, 336(6077), 95-98. https:// doi.org/10.1126/science.1216930

Konishi, M., Brown, K., Battaglini, L., \& Smallwood, J. (2017). When attention wanders: Pupillometric signatures of fluctuations in external attention. Cognition, 168(3), 16-26. https://doi.org/10.1016/j. cognition.2017.06.006

Kool, W., \& Botvinick, M. (2014). A labor/leisure tradeoff in cognitive control. Journal of Experimental Psychology: General, 143(1), 131-141. https://doi.org/10.1037/a0031048

Kool, W., \& Botvinick, M. (2018). Mental labour. Nature Human Behaviour https://doi.org/10.1038/s41562-018-0401-9

Kuhl, J. (1994). Action versus control orientation: Psychometric properties of the Action Control Scale (ACS-90). In J. Kuhl \& J. Beckmann (Eds.), Volition and personality: Action versus state orientation (pp. 47-59). Göttingen, Germany, Germany: Hogrefe.

Kuhl, J., \& Fuhrmann, A. (1998). Decomposing self-regulation and selfcontrol: The Volitional Components Inventory. In J. Heckhausen \& C. S. Dweck (Eds.), Motivation and self-regulation across the life span (pp. 15-49). New York, NY: Cambridge University Press. https://doi.org/10.1017/CBO9780511527869.003

Kurzban, R., Duckworth, A., Kable, J. W., \& Myers, J. (2013). An opportunity cost model of subjective effort and task performance. Behavioral and Brain Sciences, 36(6), 661-679. https://doi.org/10. 1017/S0140525X12003196

Laeng, B., Sirois, S., \& Gredebäck, G. (2012). Pupillometry: A window to the preconscious? Perspectives on Psychological Science, 7(1), 18-27. https://doi.org/10.1177/1745691611427305

Lavín, C., San Martín, R., \& Rosales Jubal, E. (2014). Pupil dilation signals uncertainty and surprise in a learning gambling task. Frontiers in Behavioral Neuroscience, 7, 218. https://doi.org/10. 3389/fnbeh.2013.00218

Meyniel, F., Sergent, C., Rigoux, L., Daunizeau, J., \& Pessiglione, M. (2013). Neurocomputational account of how the human brain decides when to have a break. Proceedings of the National Academy of Sciences, 110(7), 2641-2646. https://doi.org/10.1073/pnas. 1211925110

Mittner, M., Boekel, W., Tucker, A. M., Turner, B. M., Heathcote, A., \& Forstmann, B. U. (2014). When the brain takes a break: A modelbased analysis of mind wandering. The Journal of Neuroscience, 34(49), 16286-16295. https://doi.org/10.1523/JNEUROSCI.206214.2014

Murphy, P. R., O'Connell, R. G., O'Sullivan, M., Robertson, I. H., \& Balsters, J. H. (2014). Pupil diameter covaries with BOLD activity in human locus coeruleus. Human Brain Mapping, 35(8), 41404154. https://doi.org/10.1002/hbm.22466

Murphy, P. R., Robertson, I. H., Balsters, J. H., \& O'Connell, R. G. (2011). Pupillometry and P3 index the locus coeruleusnoradrenergic arousal function in humans. Psychophysiology, 48(11), 1532-1543. https://doi.org/10.1111/j.1469-8986.2011. 01226.x

Nassar, M. R., Rumsey, K. M., Wilson, R. C., Parikh, K., Heasly, B., \& Gold, J. I. (2012). Rational regulation of learning dynamics by pupil-linked arousal systems. Nature Neuroscience, 15(7), 1040 1046. https://doi.org/10.1038/nn.3130

O'Reilly, J. X., Schuffelgen, U., Cuell, S. F., Behrens, T. E. J., Mars, R. B., \& Rushworth, M. F. S. (2013). Dissociable effects of surprise and model update in parietal and anterior cingulate cortex.
Proceedings of the National Academy of Sciences, 110(38), E3660-E3669. https://doi.org/10.1073/pnas.1305373110

Pajkossy, P., Szőllősi, Á., Demeter, G., \& Racsmány, M. (2017). Tonic noradrenergic activity modulates explorative behavior and attentional set shifting: Evidence from pupillometry and gaze pattern analysis. Psychophysiology, 54(12), 1839-1854. https://doi.org/10.1111/ psyp. 12964

Peirce, J. W. (2007). PsychoPy-Psychophysics software in Python. Journal of Neuroscience Methods, 162(1-2), 8-13. https://doi.org/ 10.1016/j.jneumeth.2006.11.017

Preuschoff, K., t' Hart, B. M., \& Einhäuser, W. (2011). Pupil dilation signals surprise: Evidence for noradrenaline's role in decision making. Frontiers in Neuroscience, 5, 1-12. https://doi.org/10.3389/ fnins.2011.00115

R Core Team. (2017). R: A language and environment for statistical computing. $R$ Foundation for Statistical Computing. Vienna, Austria: R Foundation for Statistical Computing. Retrieved from www.R-project.org

Rajkowski, J., Kubiak, P., \& Aston-Jones, G. (1994). Locus coeruleus activity in monkey: Phasic and tonic changes are associated with altered vigilance. Brain Research Bulletin, 35(5-6), 607-616. https://doi.org/10.1016/0361-9230(94)90175-9

Rushworth, M. F. S., Kolling, N., Sallet, J., \& Mars, R. B. (2012). Valuation and decision-making in frontal cortex: One or many serial or parallel systems? Current Opinion in Neurobiology, 22(6), 946955. https://doi.org/10.1016/j.conb.2012.04.011

Said, C. P., \& Todorov, A. (2011). A statistical model of facial attractiveness. Psychological Science, 22(9), 1183-1190. https://doi.org/10. 1177/0956797611419169

Sara, S. J. (2015). Locus Coeruleus in time with the making of memories. Current Opinion in Neurobiology, 35(August 2015), 87-94. https:// doi.org/10.1016/j.conb.2015.07.004

Sara, S. J. (2016). Locus coeruleus reports changes in environmental contingencies. Behavioral and Brain Sciences, 39, e223. https:// doi.org/10.1017/S0140525X15001946

Sara, S. J., \& Bouret, S. (2012). Orienting and reorienting: The locus coeruleus mediates cognition through arousal. Neuron, 76(1), 130 141. https://doi.org/10.1016/j.neuron.2012.09.011

Servan-Schreiber, D., Printz, H., \& Cohen, J. (1990). A network model of catecholamine effects: Gain, signal-to-noise ratio, and behavior. Science, 249(4971), 892-895. https://doi.org/10.1126/science. 2392679

Singmann, H., Bolker, B., Westfall, J., \& Aust, F. (2018). afex: Analysis of factorial experiments. Retrieved from https://cran.r-project.org/ package $=$ afex

Steel, P. (2010). Arousal, avoidant and decisional procrastinators: Do they exist? Personality and Individual Differences, 48(8), 926-934. https://doi.org/10.1016/j.paid.2010.02.025

Unsworth, N., \& Robison, M. K. (2016). Pupillary correlates of lapses of sustained attention. Cognitive, Affective, \& Behavioral Neuroscience, 16(4), 601-615. https://doi.org/10.3758/s13415016-0417-4

Unsworth, N., \& Robison, M. K. (2018). Tracking arousal state and mind wandering with pupillometry. Cognitive, Affective, \& Behavioral Neuroscience, 18(4), 638-664. https://doi.org/10.3758/s13415018-0594-4

Urai, A. E., Braun, A., \& Donner, T. H. (2017). Pupil-linked arousal is driven by decision uncertainty and alters serial choice bias. Nature Communications, 8, 14637. https://doi.org/10.1038/ncomms14637

van der Meer, E., Beyer, R., Horn, J., Foth, M., Bornemann, B., Ries, J., ... Wartenburger, I. (2010). Resource allocation and fluid intelligence: Insights from pupillometry. Psychophysiology, 47(1), 158169. https://doi.org/10.1111/j.1469-8986.2009.00884.x

Van Slooten, J. C., Jahfari, S., Knapen, T., \& Theeuwes, J. (2018). How pupil responses track value-based decision-making during and after 
reinforcement learning. PLoS Computational Biology, 14(11), e1006632. https://doi.org/10.1371/journal.pcbi.1006632

Varazzani, C., San-Galli, A., Gilardeau, S., \& Bouret, S. (2015).

Noradrenaline and dopamine neurons in the reward/effort tradeoff: A direct electrophysiological comparison in behaving monkeys. Journal of Neuroscience, 35(20), 7866-7877. https://doi.org/10. 1523/JNEUROSCI.0454-15.2015

von der Gablentz, J., Tempelmann, C., Münte, T. F., \& Heldmann, M. (2015). Performance monitoring and behavioral adaptation during task switching: An fMRI study. Neuroscience, 285, 227-235. https://doi.org/10.1016/j.neuroscience.2014.11.024

Wood, S. N. (2017). Generalized additive models: An introduction with R. Boca Raton, FL: Chapman \& Hall/CRC. https://doi.org/10.1111/ j.1541-0420.2007.00905 3.x

Publisher's note Springer Nature remains neutral with regard to jurisdictional claims in published maps and institutional affiliations. 